\title{
Bottlenecks Identification Model of Passengers Flow through Safety Screening Process Based on Petri Net
}

\author{
Wenjie Li \\ School of North China Electric Power University, Baoding 071000, China; \\ 363378037@qq.com
}

\begin{abstract}
Keywords: Passengers Flow, Bottleneck Identification, Petri Net, Secret Private Network, Markov Chain.
\end{abstract}

\begin{abstract}
In this paper, three progressive models are established - the generic factor-determining model based on Petri Network, the asses model based on Secret Private Network (SPN), the dynamic model based on Markov Chain (MC), aiming at identifying the bottlenecks. The whole work is dependent on the calculation of the token value, the larger Token is, the more congested the process is. The fundamental elements in the Petri Net are firstly determined to help analyze procedures, then the factor-determining model based on Petri Net is built to generate the further SPN model. According to reasonable statistics, the time required for each step in the security check is calculated, which determines the initiation rate. Then the MC model of the same structure as the SPN model is established to determine the probability of reachable state, which is changed into the token value of place. Via analysis of the results, the problem area existing in current process is finally orientated - the preparation of belongings for X-ray screening.
\end{abstract}

\section{Establishment of the Model}

\section{1 (Description of the Petri Net}

There are three fundamental elements in Petri Net, which are place, transition and token. Meanings of the symbols representing the elements in the safety screening process are listed as followed:

Table.1 Definitions of Symbols in Place and Transition

\begin{tabular}{|c|c|}
\hline Symbol & Definition \\
\hline$i$ & Passenger who has arrived at the airport \\
\hline $\mathrm{P}_{1}$ & Passenger who has passed ID check \\
\hline $\mathrm{P}_{2}$ & Belongings which is ready for X-ray screening \\
\hline $\mathrm{P}_{3}$ & Belongings which has passed X-ray screening \\
\hline $\mathrm{P}_{4}$ & Belongings which has flagged for additional search \\
\hline $\mathrm{P}_{5}$ & Belonging waiting for pick \\
\hline $\mathrm{P}_{6}$ & Passengers who is ready for wave scanner or metal detector \\
\hline $\mathrm{P}_{7}$ & Passenger who has passed wave scanning \\
\hline $\mathrm{P}_{8}$ & Passenger who failed wave scanning and will receive a pat down inspection \\
\hline $\mathrm{P}_{9}$ & Passenger who is ready for collecting their belongings \\
\hline$O$ & Passenger who has departed \\
\hline$t_{1}$ & ID check \\
\hline$t_{2}$ & Preparing all their belongings for check \\
\hline$t_{3}$ & Belongings contain dangerous items \\
\hline$t_{4}$ & Additional search or screening by an officer \\
\hline$t_{5}$ & Belongings pass the X-ray screening \\
\hline$t_{6}$ & Millimeter screening or metal detecting for passenger \\
\hline$t_{7}$ & Passenger pass the wave scanning or metal detecting \\
\hline$t_{8}$ & Passenger fail the wave scanning or metal detecting \\
\hline$t_{9}$ & Pat down inspection \\
\hline$t_{10}$ & Passenger collect belongings and depart \\
\hline$t_{11}$ & \\
\hline
\end{tabular}




\subsection{Initial Model Based on Petri Net}

Via analysis, we conclude that pre-check and regular passengers go through the same security screening process, the only difference between them is that pre-check passengers spends less time than regulars during some steps, which is shown in figure 1.

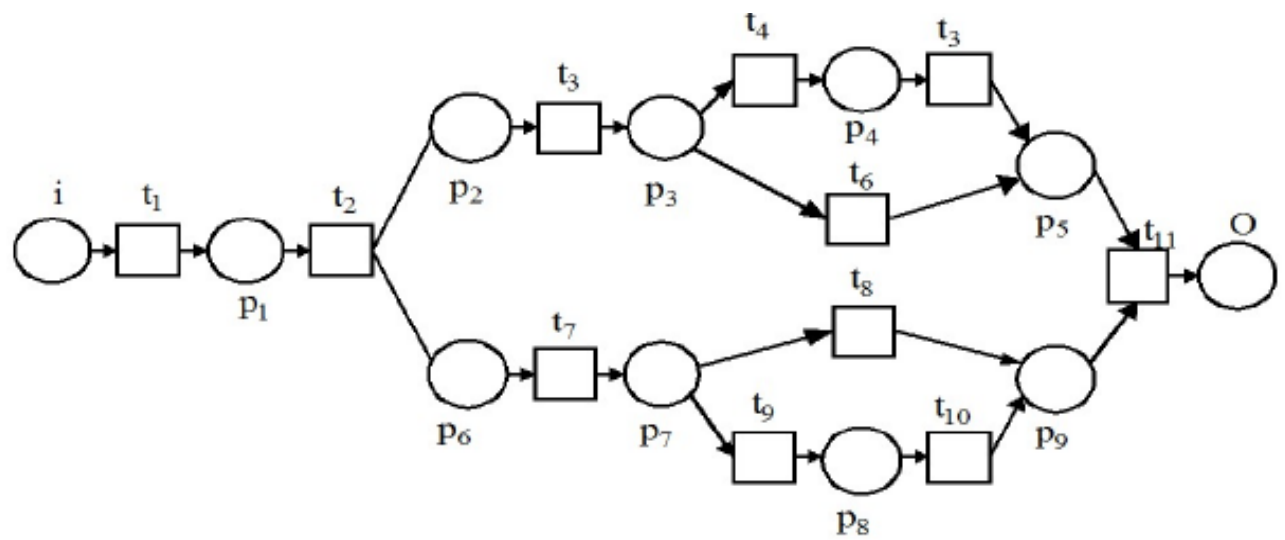

Fig 1: Petri Net of Safety Screening Process

\subsection{Implementation of the Model}

According to actual meaning of each transition, we assume that $t_{4} / t_{6} / t_{8} / t_{9}$ is an instantaneous transition, and $\alpha 1$ is the probability of $t_{4}$, then the probability of t6 is $1-\alpha 1$. In addition, the rest of the transitions get $\lambda i$ by calculating their actual time of completion.

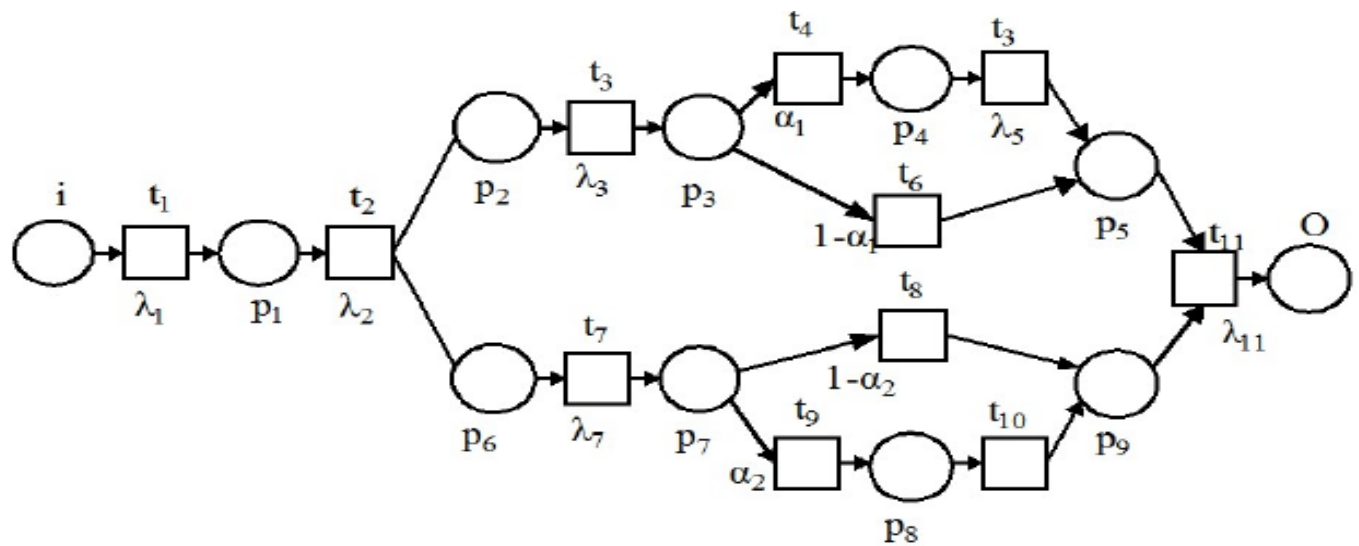

Fig.2: SPN Model of Safety Screening Model

\subsection{Establishment of the MC (Markov Chain) Model}

The reachable matrix of the SPN model can be obtained from Fig.2. Through extracting and integrating the data of the matrix, we get the MC(Markov Chain) : 


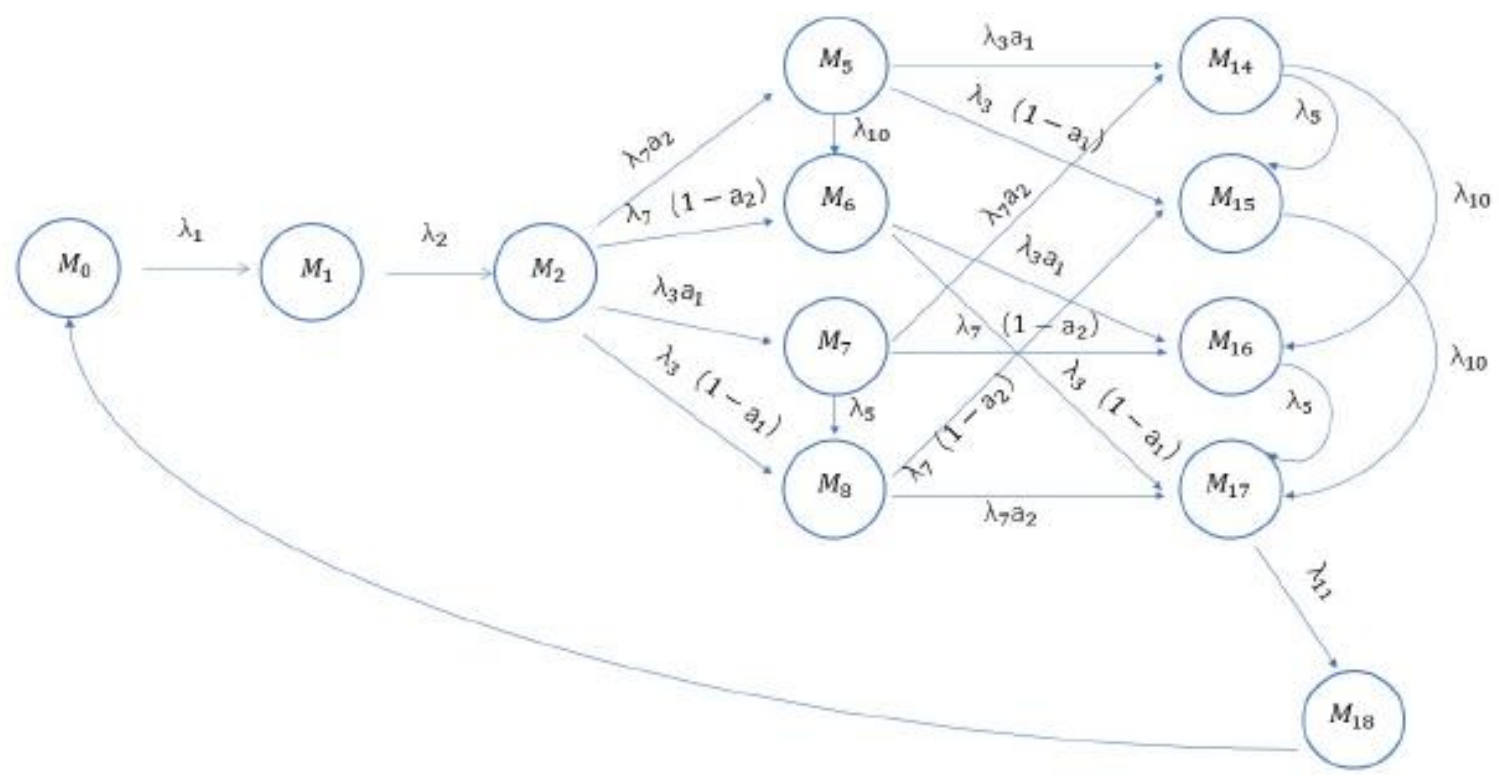

Fig.3: Markov Chain Extracted from the Matrix Above

\subsection{Solution of the Probability of Actual states in MC}

There is often one pre-check lane open for every three regular lanes, so we determine that the value of $\lambda_{3}$ and $\lambda_{7}$ is twice of the regular's rate. It's easy to get the density matrix $Q$, and use the equation $\mathrm{X}^{*} \mathrm{Q}=0$. Thus the equation of pre-check and regular passengers can be calculated as follows:

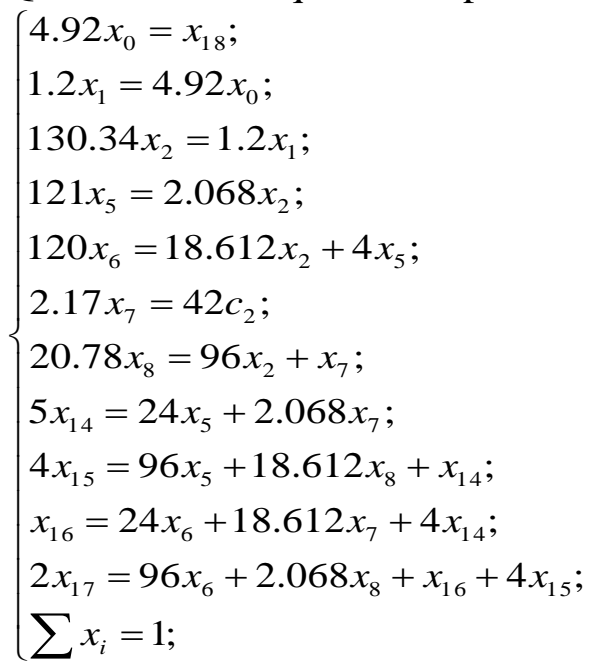

The quantity of token on average is calculated as below:

$U(P)=\sum_{k} k^{*} P[M(P)=k]$

Where $U(P)$ denotes the quantity on average. $k$ denotes the number of initial tokens contained in our model.

\subsection{Validation and Quantitative Analysis of Bottlenecks}

The average number of tokens for Re.-P1 is 0.3899, which indicates that Token number of P1 (passenger waiting for security screening) is in the larger expectations, it means that this link usually brings about bottlenecks. Generically, the most likely reasons for passengers to wait is that the time spent on preparations is too long. Meanwhile, the average number of pre-check's tokens in place P1 is much smaller than regular's, with no bottlenecks. 


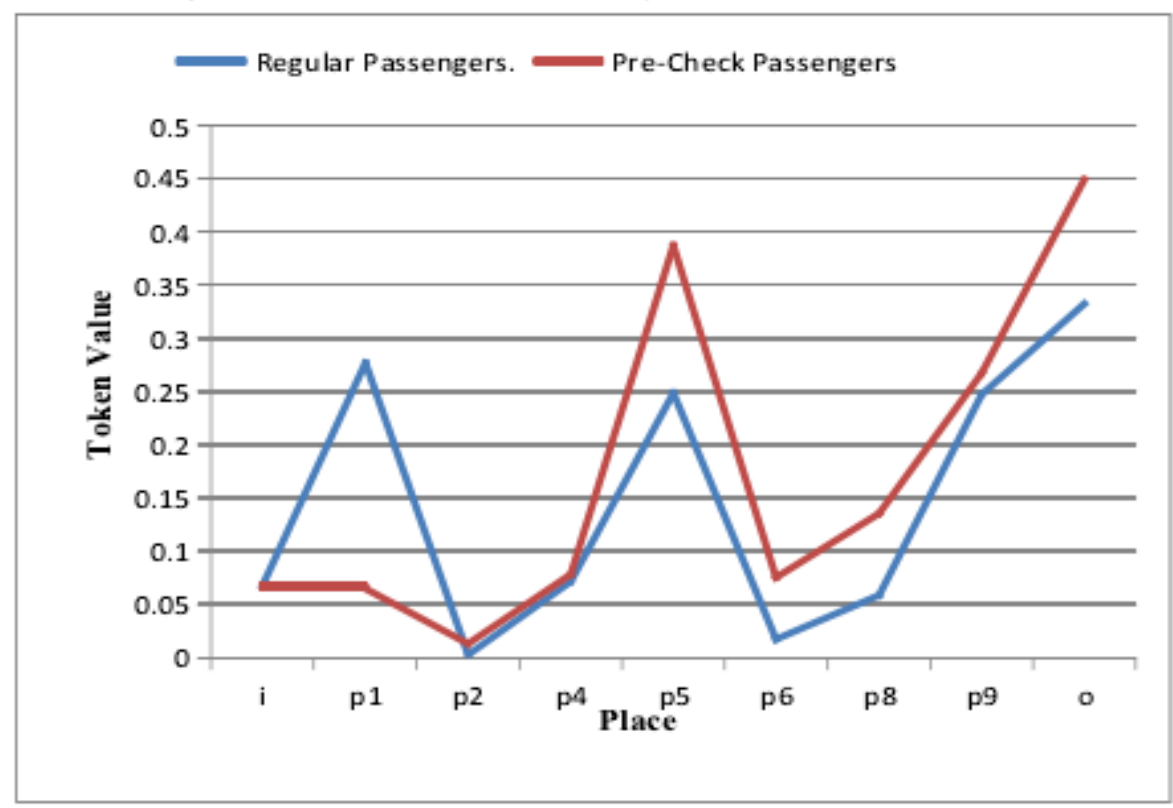

Fig.4: The Token Values of Each Link

\section{Conclusion}

In the regular lanes, there is a shortage of resources in the link with bottleneck, and the resources allocated in other links are relatively abundant, which leads to low efficiency of the security service process. Therefore, adjusting the number of security gates and the functional allocation of each security port can help eliminate bottlenecks in the security service process and help reduce queuing time in the regular channel, thus improving the performance and efficiency of the security service flow.

\section{References}

[1]. Guoyang Jia. Study on optimization of terminal passenger screening service process based on LCIOWF [D]. Harbin: Harbin Institute of Technology, 2015.

[2]. Li Z'Debashis Basu'Hamed A. Numerical Simulatin of Sidewall Effects on the Acoustic Field in Transonic Cavity[R].AIAA-2007-1456'2007.

[3]. Mccarley J S, Kram er AF, Wicken CD, et al. Visual Skills in Airport-Security Screening [J]. Psychological Science, 2004,(5);302-306(5). 